\title{
Variación de la resistencia cortante del material de relave por causas meteorológicas y su influencia en la estabilidad física de la presa de relaves $\mathrm{N} .^{\circ} 3$ Mahr Túnel
}

Fernando Mendoza Maldonado ${ }^{1}$

RECIBIDO: 18/10/2018 ACEPTADO: 04/02/2019

\begin{abstract}
RESUMEN
Los agentes meteorológicos pueden producir alteraciones en la composición física y química del material almacenado en una presa de relaves, pues modifican sus propiedades y sus condiciones de estabilidad física. En esta investigación se evaluó el comportamiento de la resistencia cortante del relave por causas meteorológicas y su influencia en la estabilidad física de la presa $\mathrm{N} .^{\circ} 3$ Mahr Túnel. Además, se ejecutaron ensayos triaxiales tipo CU en muestras inalteradas extraídas de la presa para diferentes periodos de exposición. Los resultados indican que la resistencia cortante incrementa cuando aumenta el periodo de exposición meteorológica, lo que a su vez aumenta los factores de seguridad del análisis de estabilidad de la presa.
\end{abstract}

Palabras-claves: Presas de relave; resistencia cortante; agentes meteorológicos; ensayo triaxial CU; estabilidad física.

\section{INTRODUCCIÓN}

Considerando la importancia de la industria minera peruana (producida por el potencial de reservas de minerales y la existencia de importantes proyectos mineros), es posible percibir la disposición del sector privado responsable y del país por comprometerse a desarrollar y promover una actividad minera moderna, social y ambientalmente responsable. Así, en el Perú, se han construido y operado presas de relave de gran altura y recrecimientos secuenciales a fin de contribuir con el desarrollo sostenible y generar progresos en la nación. Lo anterior exige mayores requerimientos técnicos, con el propósito de garantizar la estabilidad física de las presas, particularmente ante procesos meteorológicos que pueden relacionarse con el periodo de disposición a largo plazo, pues esto da como resultado, por ejemplo, una alta saturación de las presas, generando presiones intersticiales, factor crítico que provoca fallas en los taludes de las presas.

Los investigadores Rico, Benito, Salgueiro, et al. (2007) indican que si bien es de suponerse que las presas de relaves deben durar para siempre, la experiencia muestra que los desbordamientos representan amenazas ambientales latentes, especialmente en la etapa posterior al cierre de minas. Por otro lado, Actis (2000) señala que las propiedades físicas de los relaves pueden ser determinadas a través de ensayos de laboratorio, de fricción y/o cohesión, y por ensayos triaxiales drenados, no drenados, lentos, rápidos, consolidados y de corte directo, tomando en cuenta las condiciones de operación de la presa.

La presa de relaves $\mathrm{N}^{\circ} 3$ de la concesión de beneficio Mahr Túnel está ubicada en el distrito y provincia de Yauli, departamento de Junín, Perú, a una altitud promedio de 4000 m s. n. m y con un clima frío debido a la altitud. El área está afectada en su mayor parte por un tiempo lluvioso, con máximas precipitaciones entre noviembre y abril, asimismo persisten las nevadas y granizos. La precipitación máxima anual en época húmeda es $1188 \mathrm{~mm}$ y en periodo seco (de mayo a noviembre), $576 \mathrm{~mm}$. Además, la temperatura máxima alcanza alrededor de los $17^{\circ} \mathrm{C}$, disminuye hasta $-13^{\circ} \mathrm{C}$ en julio y desciende hasta cerca de $0{ }^{\circ} \mathrm{C}$

1 Bachiller en Ingeniería Civil, con estudios de maestría en Geotecnia, por la Universidad Nacional Mayor de San Marcos. Es consultor y supervisor de obras civiles y proyectos mineros relacionados con la ingeniería geotécnica en el Ministerio de Energía y Minas. Lima, Perú. E-mail: fmendoza@minem.gob.pe 
entre septiembre y diciembre. La temperatura media es de $5.7^{\circ} \mathrm{C}$, conforme a la información obtenida en la estación meteorológica de Pachachaca, a 4000 m s. n. m.

Es necesario mencionar que la presa $\mathrm{N} .^{\circ} 3$ ha estado expuesta a los agentes meteorológicos, los cuales han producido cambios periódicos de temperatura, congelación del agua y modificaciones en la mineralogía del relave. De la misma forma, estos fenómenos han ocasionado alteraciones en la composición física y química de la presa, causando una variación de las propiedades del relave y, en consecuencia, de su estabilidad física.

Lo anterior ha sido examinado por diversos investigadores, como Ormazábal (2008), quien realizó un estudio sobre la evaluación de la influencia del tiempo (envejecimiento) en el comportamiento estático y dinámico de la arena de relave extraída del tranque Ovejería (ubicado en La Rinconada de Ovejería, a 550 m s. n. m) en la región Metropolitana, provincia de Chacabuco, Chile.

Sobre la influencia de las causas meteorológicas, Joshi, Achari, Kaniraj y Wijiweera (1995) realizaron una investigación, a través de experimentos de laboratorio, para estudiar y registrar los efectos de envejecimiento en la arena, además de revisar la influencia de los tipos de arena y del fluido presente en los poros del suelo. Del mismo modo, Richefeu, El Youssoufi y Radjai (2006) examinaron las propiedades de resistencia al corte de materiales granulares húmedos en un estado pendular (donde la fase líquida es discontinua) como una función del contenido de agua. La arena y los envases de vidrio se humedecieron y se probaron en una célula de corte directo y bajo varias presiones de confinamiento.

\section{Justificación de la investigación}

Esta investigación busca determinar con más certeza las condiciones de estabilidad física de la mencionada presa. Por ello, se han examinado los parámetros geotécnicos del material de relleno de la misma, teniendo en cuenta su variación en el tiempo por la influencia de los agentes meteorológicos. Así, de acuerdo a los factores de seguridad (FS) obtenidos en el análisis de la estabilidad física, ha sido posible conocer las condiciones de seguridad y protección ambiental que se deben aplicar al espacio.

\section{Problema general y específico}

\subsection{Problema general}

¿Cómo evaluar el comportamiento de la resistencia cortante del material de relleno de la presa conformada por relave grueso, modificado por causas meteorológicas y temporales, y su influencia en la estabilidad de taludes de la presa de relaves $\mathrm{N} .{ }^{\circ} 3$ ?

\subsection{Problema específico}

De acuerdo al marco de investigaciones geotécnicas, ¿qué tipo de muestreo y ensayos de mecánica de suelos son adecuados para estimar la variación de los parámetros de resistencia cortante del material de relleno de la presa conformada por relave grueso, la cual se modifica por causas meteorológicas, y cuál es la influencia de esto último en la estabilidad física de la presa de relaves $\mathrm{N} .^{\circ} 3$ ?

\section{Objetivo de la investigación}

\subsection{Objetivo general}

Evaluar el comportamiento de la resistencia cortante del material de relleno conformado por relave grueso, teniendo en cuenta las causas meteorológicas y los posibles efectos en la estabilidad física de la presa de relaves $N .^{\circ} 3$.

\subsection{Objetivo específico}

Determinar la variación de la resistencia cortante del material de relleno conformada por relave grueso. Evaluar dicha alteración a partir de ensayos triaxiales obtenidos de muestras inalteradas, los cuales son representativos ante los agentes meteorológicos, y establecer su influencia en la estabilidad física de la presa de relaves $N .^{\circ} 3$.

\section{METODOLOGÍA}

\section{Recopilación y análisis de la información existente}

Consistió en la recopilación y análisis de información respecto a las condiciones de sitio, diseños de ingeniería, reportes de construcción y operación del depósito de relaves $\mathrm{N} .{ }^{\circ} 3$, las cuales fueron proporcionadas por Volcan Compañía Minera S. A. A. Además, se consiguió información relacionada a las autorizaciones del depósito en el Ministerio de Energía y Minas del Perú.

\section{Investigaciones de campo y ensayo de laboratorio}

\subsection{Investigaciones geotécnicas}

Se ejecutó un programa de investigaciones geotécnicas de campo y laboratorio, además se utilizó la información de los estudios previos indicados. Para obtener muestras inalteradas del material de relave, se ejecutaron sondeos con calicatas convenientemente ubicadas y distribuidas en la presa de relaves $\mathrm{N} .{ }^{\circ} 3$. Luego de la descripción detallada del 
material, se tomaron muestras inalteradas, las cuales fueron identificadas y protegidas para ser enviadas al laboratorio de mecánica de suelos.

\subsection{Investigaciones geotécnicas de campo}

Para obtener muestras de relave sujetas a las condiciones reales de disposición ante los agentes meteorológicos, se analizaron muestras inalteradas de la presa y se ejecutaron tres calicatas ubicadas y distribuidas en la presa de relaves. Con el fin de conseguir muestras inalteradas de relave, conforme a la profundidad registrada en la calicata, se tomó en cuenta que el material de relave esté completamente fresco y que no haya perdido humedad. Luego, se preparó la superficie marcando el contorno de lo que fue la cara superior de la muestra. Las Figuras 1 y 2 ilustran la obtención de muestras inalteradas.

De manera complementaria, se utilizó la información respecto a los sondeos geotécnicos desarrollados en la etapa de construcción y operación del depósito de relaves.



Figura 1. Corte y revestimiento con parafina de muestra. Fuente: Elaboración propia

\section{Ensayos de laboratorio}

A fin de evidenciar las condiciones reales del material de relave, se ejecutó el ensayo triaxial tipo consolidado no drenado (CU) con medición de presión de poros desarrollada en la muestra. De esta forma, esos ensayos pueden compensar los efectos de perturbación por la obtención de muestras inalteradas. Por otro lado, se consideró la disponibilidad de los reportes de los ensayos triaxiales con características similares del tipo de muestra y la presión de confinamiento. Los prototipos seleccionados se comprimieron con valores de esfuerzo de confinamiento $(\sigma \mathrm{c})$ de $1,0,2,0$ y $4,0 \mathrm{~kg} / \mathrm{cm}^{2}$. Los ensayos se realizaron en el laboratorio de mecánica de suelos de la Pontificia Universidad Católica del Perú (PUCP). Asimismo, se ejecutaron ensayos estándar (propiedades físicas) para identificar y clasificar el material de acuerdo al Sistema Unificado de Clasificación de Suelos (SUCS). El ensayo triaxial consistió en preparar probetas de suelo envueltas con una membrana que sirve para drenar y tener el control de la presión intersticial al interior de la muestra, por lo que se colocan piedras porosas en

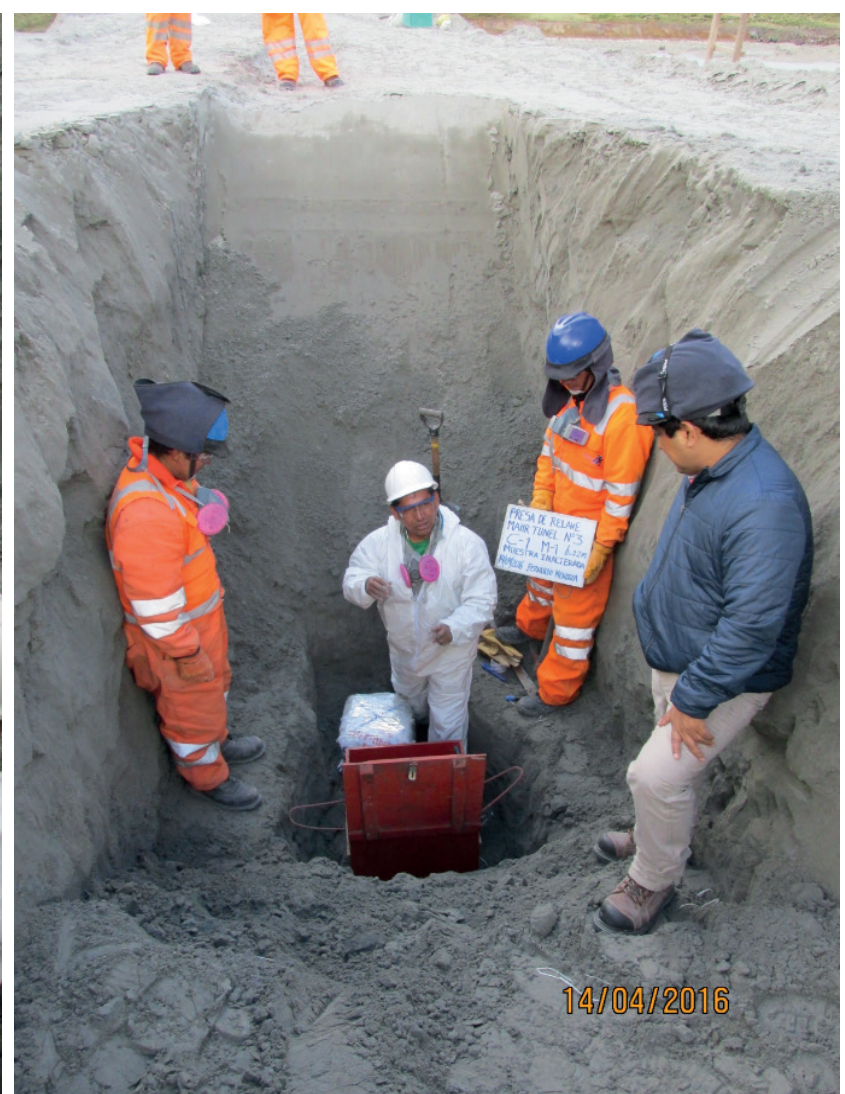

Figura 2. Empaque y transporte de muestra de relave inalterado $30 \times 30 \times 30 \mathrm{~cm}^{3}$.

Fuente: Elaboración propia 
la base y en la parte superior (González de Vallejo, Ferrer, Ortuño y Oteo, 2002).

\subsection{Trabajos de gabinete}

- Estimación de la antigüedad de los sondeos de muestras inalteradas, existentes y recientes a partir de la información topográfica, y sondeos geotécnicos desarrollados en los diversos estudios de diseño de recrecimiento de la presa de relaves $N .^{\circ} 3$, desde 2007 hasta 2016.

- Elaboración de planos de distribución de los sondeos y proyección de los periodos de exposición a los agentes meteorológicos.

- Análisis, interpretación y discusión de los resultados obtenidos de los ensayos de laboratorio triaxiales (CU) y de los ensayos estándar de las diferentes muestras, según el periodo de exposición a los agentes meteorológicos.

- Estimación de los parámetros físicos y de resistencia cortante del relave grueso a partir de los reportes de laboratorio.

- Determinación del modelo geotécnico a partir de los diferentes parámetros de los materiales y de la geometría definida para los periodos de almacenamiento.

- Análisis de estabilidad física de la presa de relaves según el modelo geotécnico definido para la etapa inicial y para los diferentes periodos de exposición meteorología.

\section{RESULTADOS}

Considerando los objetivos de esta investigación, se realizaron estudios geotécnicos de las muestras inalteradas del relave con el fin de determinar su comportamiento ante los agentes meteorológicos en el tiempo. A continuación se detallan los resultados.

\section{Estimación de los periodos de disposición de relave}

La Figura 3 presenta la estimación de antigüedad de muestras inalteradas obtenidas del sondeo de tres calicatas. El gráfico fue elaborado a partir de la información topográfica de los recrecimientos de la presa y periodo de disposición de relaves.

\section{Granulometría de los relaves}

Según los sondeos ejecutados de caracterización física y SUCS, el material de relave grueso es arena limosa (SM) y arena limosa con arcilla (SM-SC).



Figura 3. Estimación de antigüedad de muestras a partir de la curva: cota de la presa del depósito versus periodo de disposición de relaves.

Fuente: Elaboración propia. 
El contenido de las arenas (malla \#+200) está comprendido entre $57 \%$ y $83 \%$, mientras que el contenido de finos (malla \#-200) varía entre $16 \%$ y $42 \%$.

\section{Variación de los parámetros de resistencia cortante con el periodo de exposición a los agentes meteorológicos}

Los resultados de los ensayos triaxiales, en términos de esfuerzos totales, indican que cuando aumenta el tiempo de meteorización (disposición) "se incrementa" la resistencia al esfuerzo cortante no drenado. Esto significa que el tiempo de disposición ante los procesos meteorológicos influye en el incremento de la cohesión y ángulo de fricción del relave.
La Figura 4 muestra la comparación de los resultados del ángulo de fricción. Así, el ejemplar BH4SMR3 presenta un periodo de disposición estimado en 3 años, alcanzando un incremento de $60 \%$ respecto del año inicial de disposición "cero". Le sigue una tendencia creciente hasta el año 6 de disposición. En la muestra C-2 M-1 se observa un aumento de hasta $200 \%$. En el caso de la muestra BH5-SMR3, para el año 7, se observa un descenso de hasta $68 \%$ respecto del año cero.

La Figura 5 muestra la comparación de los resultados de la cohesión. Para el año cero, en la muestra SPT CM-11 se observa que la cohesión es de 0 kg/

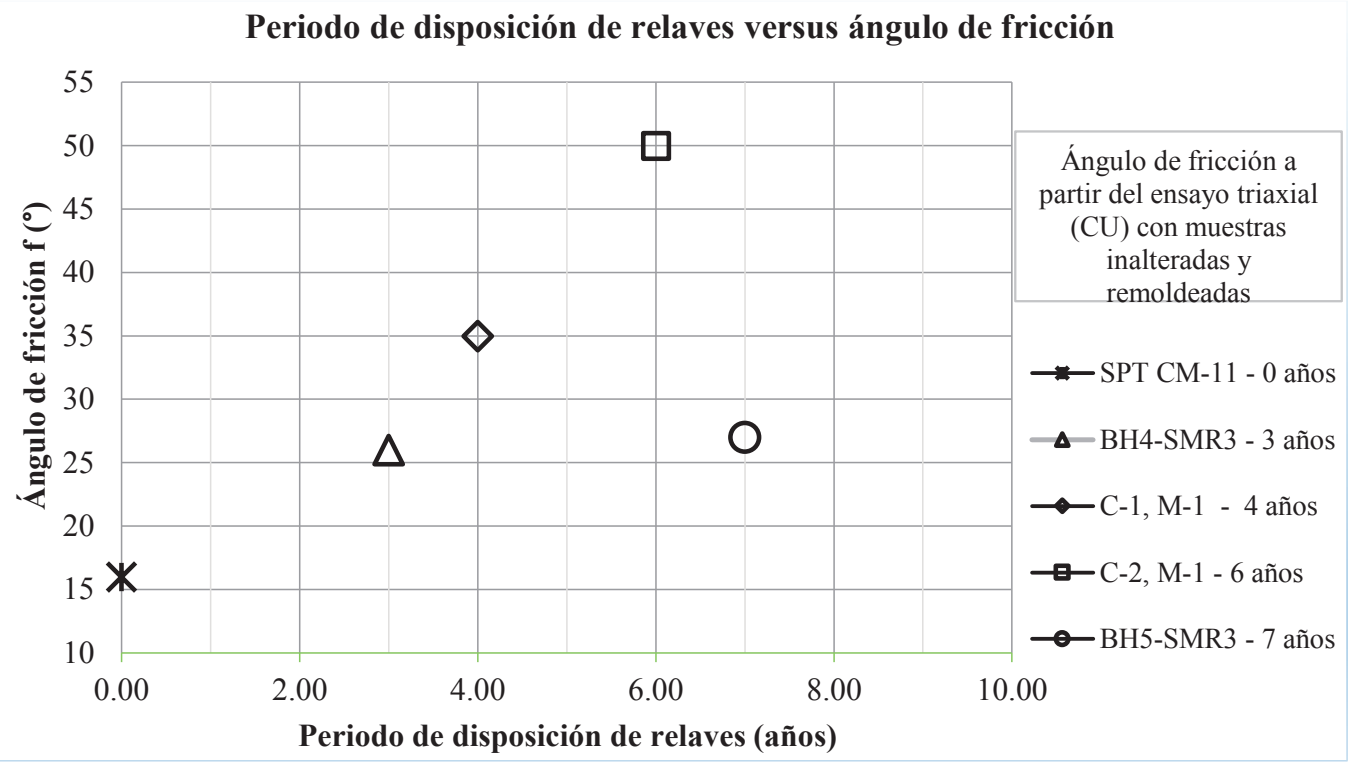

Figura 4. Variación del ángulo de fricción de relave grueso en términos de esfuerzos totales.

Fuente: Elaboración propia.

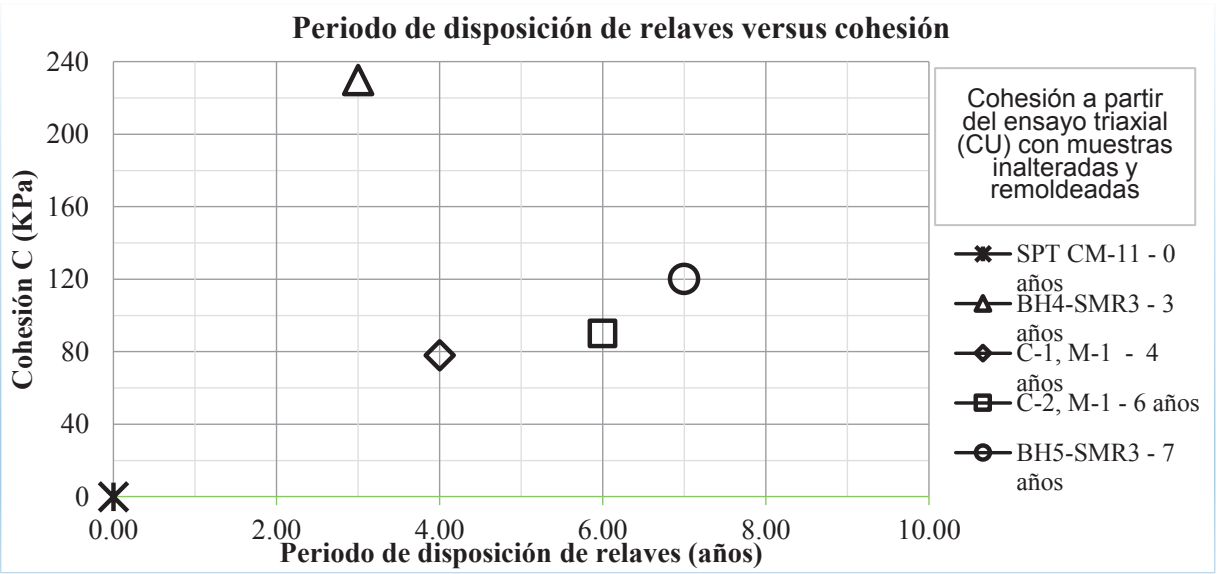

Figura 5. Variación de la cohesión del relave grueso en términos de esfuerzos totales.

Fuente: Elaboración propia. 
$\mathrm{cm}^{2}$; mientras que en la muestra BH4-SMR3, con 3 años de disposición, hay un incremento súbito que alcanza una cohesión de $230 \mathrm{~kg} / \mathrm{cm}^{2}$. Sin embargo, para el año 4, en la muestra C-1 M-1 se aprecia un descenso hasta alcanzar un valor de cohesión de $78 \mathrm{~kg} / \mathrm{cm}^{2}$. Para los siguientes años ocurre un incremento progresivo de la cohesión.

\section{Análisis de estabilidad física}

\subsection{Generalidades}

Para el análisis de la estabilidad de taludes de la presa de relaves $\mathrm{N} .{ }^{\circ} 3$ se tomó en cuenta lo siguiente:

- Se desarrolló la sección transversal de análisis en base a la configuración geométrica actual de la presa y de las perforaciones diamantinas, con lo cual se estimó la distribución de los materiales.

- La sección geométrica representa las condiciones críticas de la presa, es decir, el mayor el ángulo del talud, la carga por acción del sismo, las condiciones de nivel freático, entre otras.

- Se utilizó la metodología de equilibrio límite para evaluar la resistencia al deslizamiento de un talud, tomándose en cuenta ciertas hipótesis respecto al mecanismo de falla, la condición de equilibrio, la superficie del nivel freático, los parámetros de resistencia cortante del material, etc.

\subsection{Metodología del análisis realizado}

- Se ha utilizado el software SLIDE (Rocscience versión 6) para modelar la configuración geométrica del talud interactivamente $y$ definir los diferentes materiales, tomando en cuenta sus propiedades geotécnicas (físicas y mecánicas).

- Para determinar los factores de seguridad (FS), se partió de lo planteado por Quispe (2015), quien menciona que la definición "se lleva a cabo de manera bidimensional usando el concepto de equilibrio límite, aproximando el problema a un estado de deformación plana" (p. 4). Este autor, además, precisa que la superficie de falla crítica es aquella que proporciona el menor FS y que se encuentra de forma interactiva modificando las condiciones de búsqueda de la misma, así como evaluando las superficies de falla circular.

- A su vez, como hipótesis del análisis de la estabilidad "se considera que las propiedades [físicas y mecánicas] de los materiales que conforman las estructuras analizadas son homogéneas e isotrópicas y que el colapso se produciría como resultado de fallas simultáneas a lo largo de la superficie de deslizamiento" (Quispe, 2015, pp. 4-5). El análisis de estabilidad pseudo-estático (PE) somete una masa deslizable de dos dimensiones a una aceleración horizontal igual al coeficiente sísmico multiplicado por la aceleración de la gravedad.

- El método usado para los análisis PE es el indicado por Hynes-Griffin y Franklin (1984), el cual requiere la reducción de las propiedades de la resistencia cortante del material hasta un $20 \%$ para aquellos materiales sujetos a un incremento de la presión de poros en condiciones cíclicas (terremotos).

\subsection{Factor sísmico de diseño}

El parámetro de diseño especificado para la zona de estudio es una aceleración máxima de $0,3 \mathrm{~g}$, valor que tiene una probabilidad de excedencia de $10 \%$ en 50 años de exposición sísmica y correspondiente a un evento de 475 de periodo de retorno. EI valor recomendado para un coeficiente adecuado PE varía entre $1 / 2$ a $1 / 3$ veces del valor de la aceleración pico de terreno. En el estudio de Rennat y Miller (1997) se utilizó un coeficiente sísmico de $0,15 \mathrm{~g}$, valor que es $1 / 2$ de la aceleración máxima esperada de $0,3 \mathrm{~g}$.

\subsection{Nivel freático}

Los niveles freáticos registran profundidades de $16,20 \mathrm{~m}$ y $14,60 \mathrm{~m}$ a partir de la superficie de nivelación. Estos niveles de agua actúan como un nivel piezométrico, pues afectan principalmente al suelo de cimentación y al basamento rocoso, lo cual constituye una hipótesis conservadora, ya que el nivel de agua no es continuo. En efecto, Rodríguez y Oldecop (2011) mencionan que el agua infiltrada en la presa de relaves satura rápidamente los poros y parcialmente los llena con agua capilar. Además, la aproximación del agua freática al talud exterior de la presa tiene efectos desestabilizantes.

\section{Parámetros geotécnicos}

En la Tabla 1 se muestran las propiedades geotécnicas de los diferentes materiales utilizados en el análisis de la estabilidad. Estos parámetros de resistencia cortante se obtuvieron a partir de los ensayos triaxiales $\mathrm{CU}$ con muestras inalteradas y alteradas. 
Tabla 1. Resumen de propiedades geotécnicas de materiales.

\begin{tabular}{|c|c|c|c|c|c|c|c|}
\hline \multirow{2}{*}{ Material } & \multirow{2}{*}{$\begin{array}{c}\text { Año de } \\
\text { disposición }\end{array}$} & \multirow{2}{*}{$\begin{array}{c}\mathbf{Y}_{\mathrm{sec}}(\mathrm{kN} / \\
\mathrm{m})\end{array}$} & \multirow{2}{*}{$\mathrm{Y}_{\text {sat }}\left(\mathrm{kN} / \mathrm{m}^{3}\right)$} & \multicolumn{2}{|c|}{ Totales } & \multicolumn{2}{|c|}{ Efectivos } \\
\hline & & & & $\mathrm{C}(\mathrm{kPa})$ & $\Phi\left({ }^{\circ}\right)$ & $\mathrm{C}(\mathrm{kPa})$ & $\varphi\left({ }^{\circ}\right)$ \\
\hline Relave grueso remoldeado $(\mathrm{C} 11, \mathrm{M}-1)$ & 0 & 16 & 17,5 & 0 & 16 & 30 & 31,5 \\
\hline Relave grueso (BH4-SMR3-04) & 3 & 21 & 24 & 230 & 26 & 0 & 41 \\
\hline Relave grueso $(\mathrm{C}-1, \mathrm{M}-1)$ & 4 & 19 & 21 & 78 & 35 & 50 & 35 \\
\hline Relave grueso $(\mathrm{C}-2, \mathrm{M}-1)$ & 6 & 18.5 & 20 & 90 & 51 & 120 & 52 \\
\hline Relave grueso (BH5-SMR3-04) & 7 & 17 & 24 & 120 & 27 & 29 & 36,4 \\
\hline Relave fino $\left(^{*}\right)$ & - & 16,5 & 20,0 & 0 & 25 & - & - \\
\hline Cimentación & - & 19 & 20 & 100 & 36 & 10 & 36 \\
\hline
\end{tabular}

$\mathrm{V}_{\text {tot }}$ : peso específico total del suelo; $\mathrm{V}_{\text {Sat }}$; peso específico saturado; $C$ : cohesión; $\varphi$ : ángulo de fricción interna.

(*) Valor estimado a partir de estudios anteriores.

Fuente: Elaboración propia.

\section{Resultados del análisis de estabilidad de taludes}

Los análisis de estabilidad de la presa se realizaron considerando el relave grueso sin exposición, año cero y expuesto a los agentes meteorológicos. Para el primer caso, se han obtenido valores de FS de 1,014 en condición pseudo-estática (ver Figura 6). Para el relave con exposición a los agentes meteorológicos, se han obtenido valores de FS de 1,060 en condición pseudo-estática (ver Figura 7).

\section{DISCUSIÓN}

\section{Granulometría de los relaves}

Peters (2004) señala que la granulometría de los relaves es el reflejo del proceso de tratamiento utilizado para la obtención del mineral, el sistema de colocación y el método del vertido. Las playas de vertido tienen cierta pendiente hacia el vaso donde se depositan los relaves finos, dicha pendiente es de $0,5 \%$ a $3,0 \%$ en los primeros $30 \mathrm{~m}$, luego se suaviza hasta alcanzar valores del orden de $0,1 \%$ en las proximidades del estanque. En relaves gruesos, las pendientes medias pueden llegar hasta $4 \%$ y $6 \%$. En tamaño, los relaves son materiales generalmente superiores a $0,074 \mathrm{~mm}$, esto significa que menos del $50 \%$ son más finos que ese tamaño. Además, las lamas son "el material predominantemente del tamaño del limo, por lo cual, más del $50 \%$ y menor de 0,074 mm" (Torres, 2014, p. 42).

2. Variación del esfuerzo desviador y presión de poros respecto a la deformación de las muestras de relave con el periodo de exposición de agentes meteorológicos

La Figura 8 muestra la comparación entre los resultados del esfuerzo desviador y la deformación para

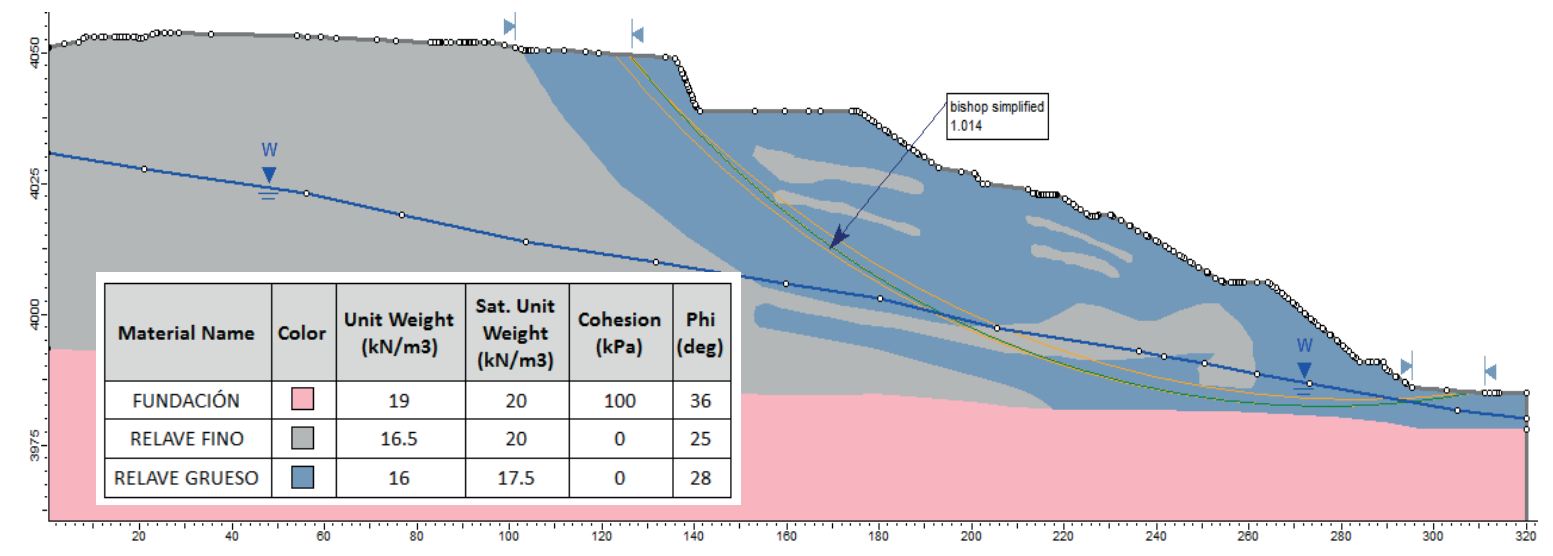

Figura 6. Análisis de estabilidad de la presa de relaves $N{ }^{\circ} 3$. Análisis PE, sin exposición meteorológica (año 0), falla circular.

Fuente. Elaboración propia. 


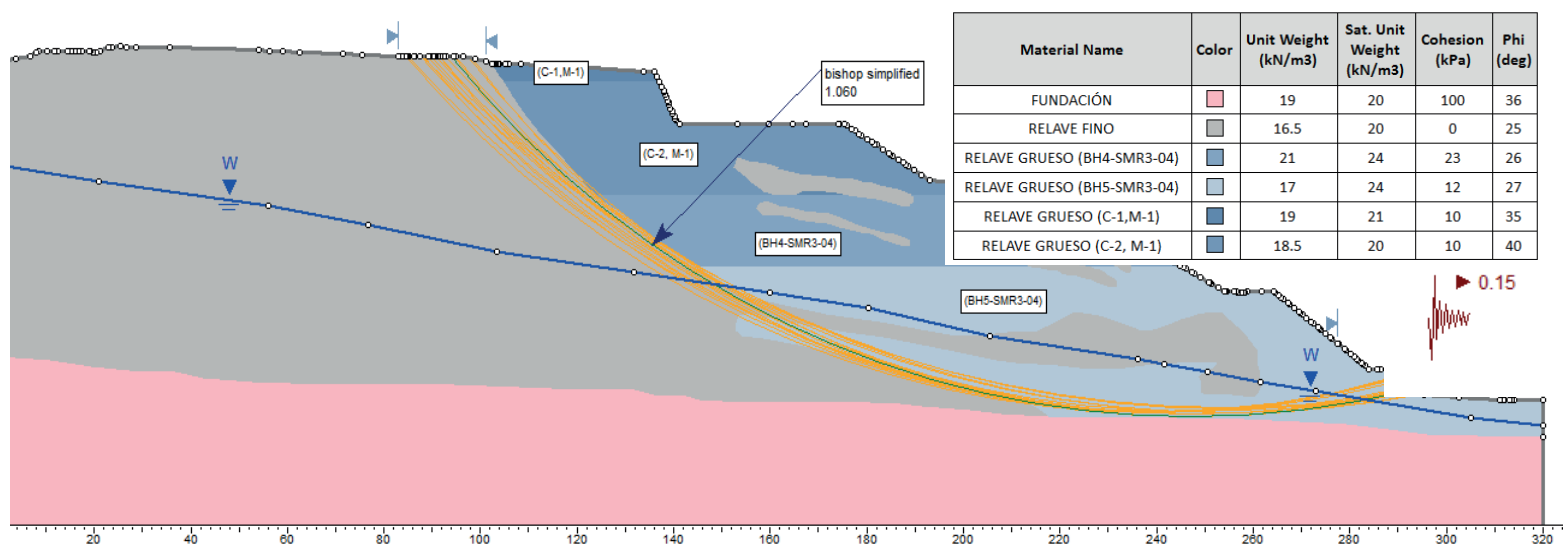

Figura 7. Análisis de estabilidad de la presa de relaves N. ${ }^{\circ}$. Análisis PE, con exposición meteorológica, falla circular.

Fuente: Elaboración propia.

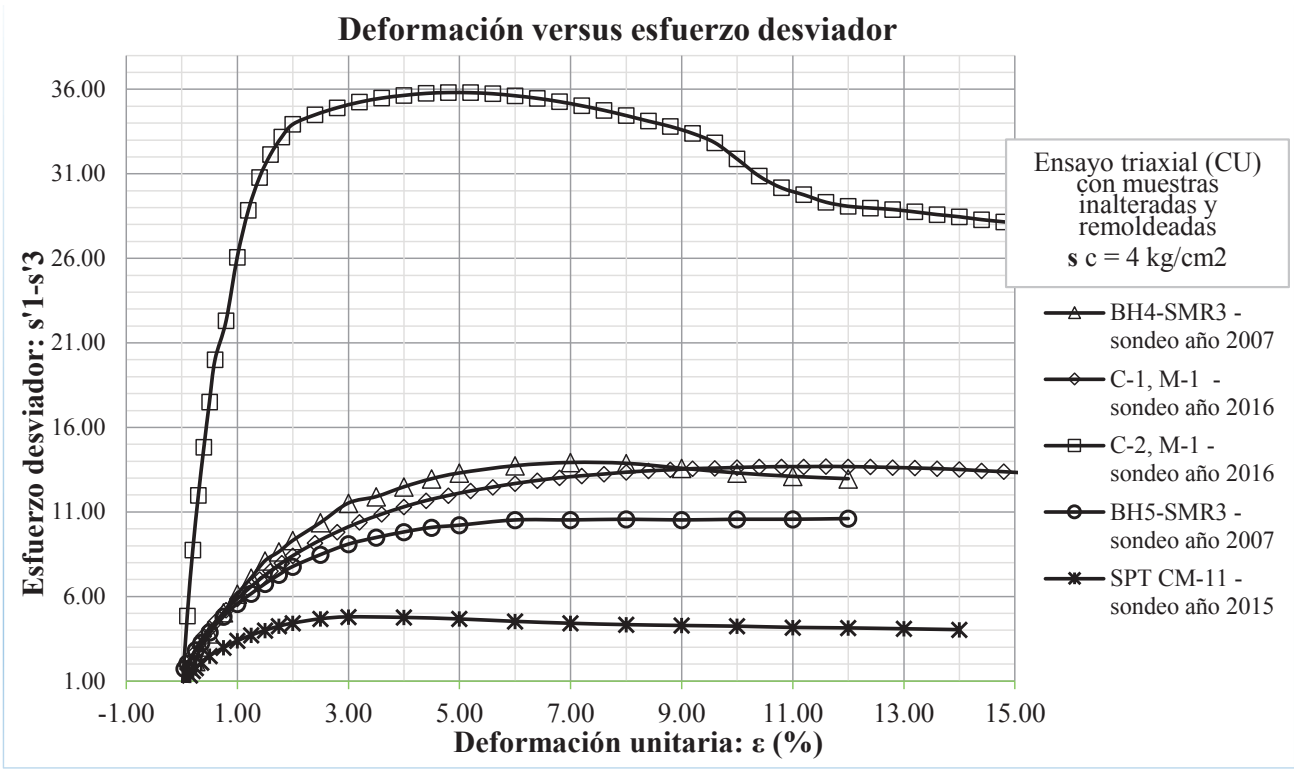

Figura 8. Deformación unitaria $(\varepsilon)$ versus esfuerzo desviador de la muestra remoldeada y muestras inalteradas ensayadas en diferentes periodos.

Fuente: Elaboración propia.

las diferentes muestras analizadas, cuya presión de cámara aplicada es de $4 \mathrm{~kg} / \mathrm{cm}^{2}$, teniendo en cuenta la estimación de los años de disposición de relaves ante procesos meteorológicos en la presa $\mathrm{N} .^{\circ} 3$.

Para el caso de la muestra (alterada) que representa el año cero, la falla es de tipo frágil, porque, al aumentar el esfuerzo, la deformación crece y tiende a un valor límite que se conserva, a pesar de que la deformación siga creciendo hasta valores muy grandes. Para las muestras inalteradas (años 3, 4, 6 y 7 de disposición de relave), aumenta el esfuerzo respecto al año cero, lo cual corresponde a una falla de tipo frágil, es decir, cuando el esfuerzo llega a un máximo, disminuye conforme la deformación au- menta, pero al crecer la deformación, el valor último tiende a ser el mismo. En el caso de la muestra que representa el año 6 (C-2 M-1), el gráfico esfuerzo versus deformación corresponde a la arena cementada, en otras palabras, la disminución rápida del esfuerzo a partir del valor máximo ocurre al crecer la deformación.

La Figura 9 muestra la presión de cámara aplicada $\sigma_{3}$, para las diferentes muestras inalteradas y remoldeadas, la resistencia es soportada inicialmente por el agua, de allí empieza el aumento de la presión de poros. Para la muestra remoldeada SPT $\mathrm{CM}-11$, la presión de agua soporta en todo momento a la resistencia del material de relave. La presión 


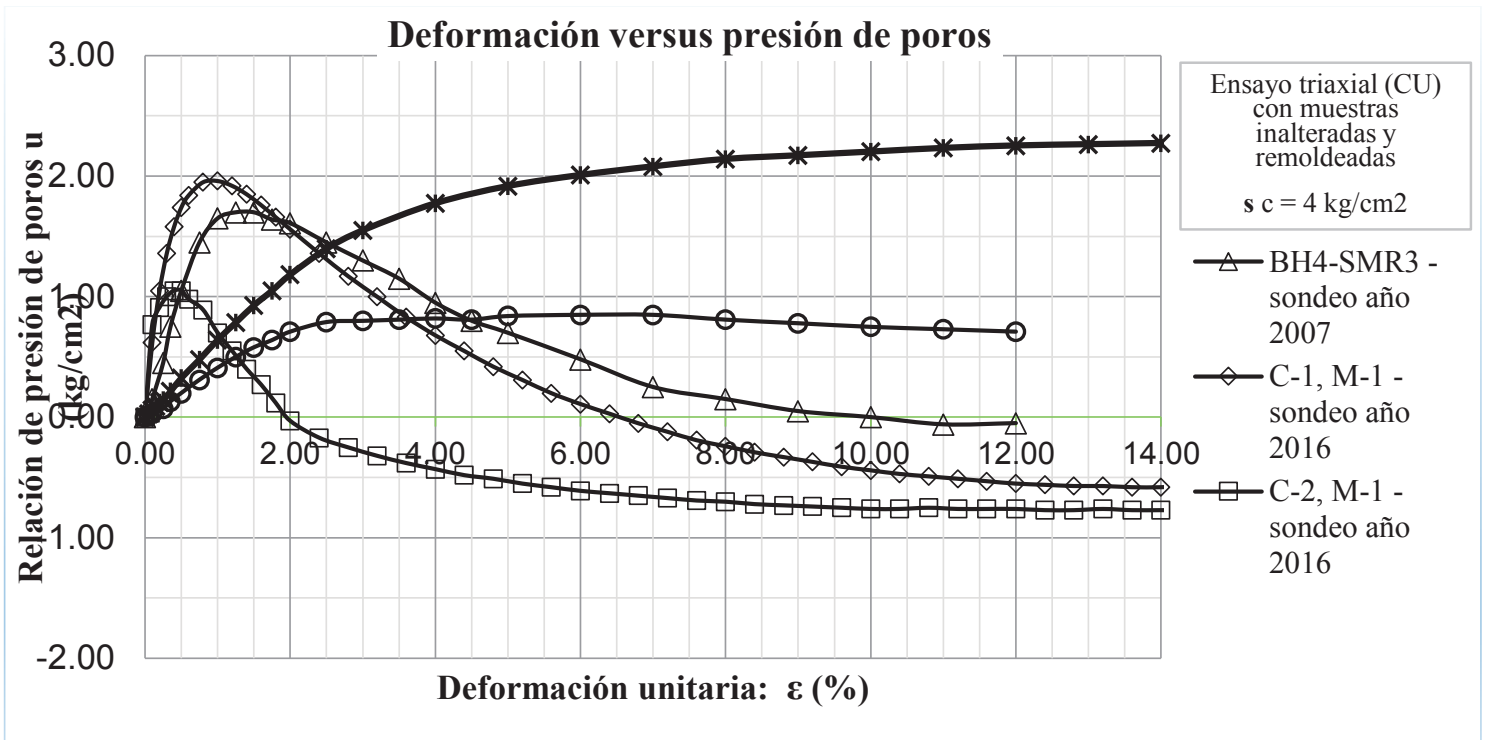

Figura 9. Deformación unitaria $(\varepsilon)$ versus presión de poros $(u)$ de las muestras inalteradas ensayadas en diferentes periodos y de la muestra remoldeada.

Fuente: Elaboración propia.

de poros se vuelve negativa en la muestra $\mathrm{BH} 4-$ SMR3, a partir de la deformación unitaria $10.0 \%$; en la muestra C-1 M-1, a partir de la deformación $6,5 \%$; y en la muestra C-2 M-1, a partir de la deformación $2.0 \%$. Esta disminución y cambio de signo en la presión de poros significa que la carga está siendo soportada por las partículas de relave y que aumenta la tensión efectiva del relave.

3. Variación de la densidad del relave con el periodo de exposición meteorológica y su influencia con los parámetros de resistencia cortante

De acuerdo a las muestras estudiadas en diferentes periodos de disposición del relave grueso, la densidad natural, para el año cero ("0") es de 1,77 $\mathrm{g} / \mathrm{cm}^{3}$; para el año 3 es de 2,33 $\mathrm{g} / \mathrm{cm}^{3}$; para el año 4 es de 2,18 g/ $/ \mathrm{cm}^{3}$; para el año 6 es de 2,02 g/ $/ \mathrm{cm}^{3}$; y para el año 7 es de $2,06 \mathrm{~g} / \mathrm{cm}^{3}$. Como se indicó, la densidad inicial (año cero) es baja; sin embargo, según pasan los años de disposición del relave, se incrementan las densidades. De la misma forma ocurre con los parámetros de resistencia cortante, pues estos se intensifican debido a la consolidación secundaria. Sobre ese punto, Badillo y Rodríguez (2005) señalan que la consolidación secundaria se atribuye generalmente al deslizamiento progresivo referido al tiempo entre las partículas del material que se reacomodan y se obtienen en un estado más compacto para adaptarse a la nueva condición de carga. Entonces, el grado de consolidación influye notablemente en las propiedades de los re- laves, los hace menos deformables, más densos y resistentes. Además, resaltan las variaciones en la densidad seca durante la consolidación con la reducción del índice de poros, lo cual lleva a la formación de estructuras más compactas y rígidas. En algunos casos, los relaves mineros presentan una importante consolidación secundaria (sin aumento de carga vertical), bien por disgregación de partículas o por fenómenos de fluencia viscosa (Ayala y Rodríguez, 1986).

\section{Factores de seguridad del análisis de estabilidad}

Según los resultados de análisis de estabilidad de la presa, los FS para la presa de relaves $\mathrm{N} .^{\circ} 3$, con el material de relave expuesto a la meteorización, son más estables en condición PE.

\section{CONCLUSIONES}

- Se evaluó el comportamiento de la resistencia cortante del material de relleno conformado por relave grueso, además, se tuvo en cuenta las causas meteorológicas y los posibles efectos en la estabilidad física de la presa de relaves $\mathrm{N} .{ }^{\circ} 3$. Considerando ese fin, se realizaron sondeos para obtener muestras inalteradas del material de relave y se siguieron los procedimientos normalizados. Luego de la extracción de las muestras, se desarrollaron y validaron los ensayos triaxiales tipo $\mathrm{CU}$ con medición de 
la presión de poros. Estos ensayos reflejaron las características de esfuerzo-deformación y resistencia del suelo por las circunstancias críticas de la presa de relave, por los efectos de perturbación de la muestra inalterada y, por ende, de los efectos meteorológicos.

- Los resultados del ángulo de fricción $(\varphi)$ obtenidos del relave grueso, en diferentes periodos de exposición a la meteorización de la presa de relaves $\mathrm{N} .^{\circ} 3$, en términos de esfuerzos totales y efectivos, fueron los siguientes: para el año $4,35^{\circ}$, y para el año 6 , $52^{\circ}$ y $51^{\circ}$. De acuerdo a la información previa, para el relave sin exposición meteorológica (año 0 ) fue de $16,5^{\circ}$ y $31,5^{\circ}$, para el año 3 fueron $26^{\circ}$ y $41,4^{\circ}$, y para el año 7 fueron $27^{\circ}$ y $36,4^{\circ}$. Esta obtención indica que el ángulo de fricción aumentó conforme se incrementó el periodo de exposición a los agentes meteorológicos.

- Los resultados de la cohesión (c) del relave grueso obtenidos en diferentes periodos de exposición a los agentes meteorológicos, en términos de esfuerzos totales y efectivos, fueron los siguientes: para el año $4,50 \mathrm{kPa}$ y $78 \mathrm{kPa}$, y para el año $6,90 \mathrm{kPa}$ y 120 $\mathrm{kPa}$. De acuerdo a la información previa sobre la presa de relaves $\mathrm{N} .{ }^{\circ} 3$, para el año 0 fueron $0 \mathrm{kPa}$ y $3 \mathrm{kPa}$, para el año 3,230 $\mathrm{kPa}$ y $0 \mathrm{kPa}$, y para el año $7,120 \mathrm{kPa}$ y 29 $\mathrm{kPa}$. Esta obtención indica que la cohesión aumentó conforme se incrementó el periodo de exposición a los agentes meteorológicos.

- La densidad natural también obtuvo resultados: para el año cero "0" resultó $1,77 \mathrm{~g} / \mathrm{cm}^{3}$, para el año 3 resultó $2,33 \mathrm{~g} / \mathrm{cm}^{3}$, para el año 4 resultó $2,18 \mathrm{~g} / \mathrm{cm}^{3}$, para el año 6 resultó 2,02 $\mathrm{g} / \mathrm{cm}^{3}$ y para el año 7 resultó $2,06 \mathrm{~g} / \mathrm{cm}^{3}$. En otras palabras, para el año inicial la densidad es baja, y - según incrementan los años de exposición a los agentes meteorológicosaumentan las densidades; por lo tanto, los parámetros de resistencia cortante se deben a la consolidación secundaria. Por ello mismo, Das (2013) señala que la consolidación secundaria del suelo se produce luego de la disipación total del exceso de presión del agua intersticial, cuando se lleva a cabo alguna deformación de la muestra por al reajuste plástico del suelo.

- A partir del incremento de la cohesión en materiales básicamente cohesivos en los relaves ensayados (arena con contenido de finos de $21,99 \%$ en promedio, es decir, porcentaje de finos bajo malla \#200), se determinó que la cohesión se incrementó. Esto produjo una progresiva cementación del material a causa del contenido mineralógico del relave, ocasionando presiones intergranulares por los efectos capilares, produciendo además una "cohesión aparente". Vale añadir que, según Lambe y Whitman (1991), la cohesión aparente se pierde en cuanto el suelo se sumerge bajo el nivel freático.

- Para el relavegruesosin exposición a los agentes meteorológicos se obtuvo un FS de 1,014 en condición pseudo-estática (PE) y para el relave con exposición se obtuvo un FS de 1,060 en condición PE. Es decir, los FS en condición expuesta a estos agentes son mayores que los FS sin exposición. Estos valores indican que la presa de relaves $\mathrm{N} .{ }^{\circ} 3$, con el material de relave expuesto a la meteorización, es más estable en condición PE.

\section{AGRADECIMIENTOS}

A los ingenieros Enrique Guadalupe Gómez, Víctor Abel Tolentino Yparraguirre y Jorge Escalante Contreras, catedráticos de la Universidad Nacional Mayor de San Marcos, por su constante apoyo en la revisión y por las correcciones acertadas. Asimismo, a Volcan Compañía Minera S. A. A., por facilitar sus instalaciones para el estudio pertinente.

\section{REFERENCIAS BIBLIOGRÁFICAS}

[1] Ayala, F. y Rodríguez, J. (1986). Manual para el diseño y construcción de escombreras y presas de residuos mineros. Madrid, España: Instituto Geológico y Minero de España.

[2] Actis, R. A. (2000). Diques de colas mineras. Cálculo, diseño, construcción y operación. Córdoba, Argentina: Fundación EMPREMIN.

[3] Badillo, J. y Rodríguez, R. (2005). Fundamentos de Mecánica de Suelos (tomo 1). México D. F., México: Limusa Noriega Editores.

[4] Das, B. M. (2013). Fundamentos de ingeniería geotécnica. México D. F., México: Cengage Learning Latinoamérica.

[5] González de Vallejo L., Ferrer M., Ortuño L. y Oteo, C. (2002). Ingeniería geológica. Madrid, España: Pearson Education.

[6] Hynes-Griffin, M. E. y Franklin, A. G. (1984), Rationalizing the Seismic Coefficient Method. Recuperado de http://acwc.sdp. 
sirsi.net/client/en_US/default/index.assetbox. assetactionicon.view/1003264

[7] Joshi, R. C., Achari, G. Kaniraj, S. R. y Wijiweera, H. (1995). Effect of aging on the penetration resistance of sands. Canadian Geotechnical Journal, 32(5), 767-782.

[8] Lambe, T. W. y Whitman, R. V. (1991). Mecánica de fluidos. Mexico D. F., Mexico: Limusa Noriega Editores.

[9] Ormazábal, M. G. (2008). Estudio en laboratorio del efecto envejecimiento en el comportamiento de arenas de relave. (Tesis de pregrado). Universidad de Chile, Santiago de Chile. Recuperado de http://repositorio.uchile. cl/bitstream/handle/2250/104855/ormazabal_ $\mathrm{ml}$.pdf? sequence $=3$ \&isAllowed $=y$

[10] Peters, G. (2004). Propiedades geotécnicas de arenas de relave y sus implicancias en el diseño de tranques. En E. Riesco (Presidencia), $\checkmark$ Congreso Chileno de Ingeniería Geotecnia. Conferencia llevada a cabo en la Universidad de Chile, Santiago de Chile.

[11] Quispe, A. (2015). Factor de seguridad en la estabilidad en pad de lixiviación (Tesis de pregrado). Universidad Nacional de Ingeniería, Lima. Recuperado de http://cybertesis.uni.edu. pe/handle/uni/5606
[12] Rodríguez, R. y Oldecop, L. (2011). Humedad y estabilidad geotécnica de presa de relaves. Recuperado de https://www.researchgate. net/publication/306292392_Humedad_y_ estabilidad_geotecnica_de_presas_de_ relaves.

[13] Rico, M., Benito, G., Salgueiro, A., et al. (2007). Reported tailing dam failures: A review of the European incidents in the worldwide context. Journal of Hazardous Materials, 152(2), 846852.

[14] Rennat, E. y Miller, S. (1997). Guía ambiental para la estabilidad de taludes de depósitos de desechos sólidos de mina. Lima, Perú. Ministerio de Energía y Minas. Recuperado de http://www.minem.gob.pe/minem/archivos/file/ DGAAM/guias/guiaestabilidad.pdf

[15] Richefeu, V., El Youssoufi, M. S. y Radjaï, F. (2006). Shear strength properties of wet granular materials. Physical Review, (73), 1-12. Recuperado de https://www.researchgate. net/publication/6981623_Shear_strength_ properties_of_wet_granular_materials.

[16] Torres, E. (2014). Práctica supervisada. Proyecto de sistema de sedimentación para la compañía minera El Aguilar. Recuperado de https://rdu.unc.edu.ar/handle/11086/1533. 


\section{Shear strength variation of tailings material due to meteorological causes and its influence on the physical stability of tailings dam No. 3 Mahr Túnel}

Fernando Mendoza Maldonado ${ }^{1}$

Received: 18/10/2018 AcCepted: 04/02/2019

\begin{abstract}
Meteorological conditions can produce alterations in the physical and chemical composition of materials stored in a tailings dam, as they modify their properties and the dam's slope stability conditions. In this study, the shear strength behavior of tailings, as a result of meteorological causes, and its influence on the slope stability of tailings dam No. 3 Mahr Túnel, was assessed. To this end, consolidated undrained (CU) triaxial tests were conducted on undisturbed samples retrieved from the dam for different periods of weather exposure. The results show that tailings shear strength increases as the weather exposure period increases, which in turn enhance the factors of safety of the tailings dam stability analysis.
\end{abstract}

Keywords: Tailings dams; shear strength; meteorological conditions; CU triaxial test; physical stability.

\section{INTRODUCTION}

Considering the importance of the Peruvian mining industry (due to potential mineral reserves and major existing mining projects), it is possible to observe the willingness of both the responsible private sector and the country to commit to the development and promotion of modern, socially and environmentally responsible mining. Thus, both high-rise and sequentially raised tailings dams have been built and operated in Peru, in order to contribute to sustainable development and to promote progress in the nation. This demands greater technical requirements to guarantee slope stability of dams, especially with regard to meteorological processes that may be related to the long-term disposal period, as this generates, for example, high saturation in dams, which generates pore pressure, a critical factor that causes dam slope failures.

Researchers Rico, Benito, Salgueiro, et al. (2007) indicate that while tailings dams are supposed to last forever, past experience shows that spills are a latent environmental threat, particularly after mine closure. Additionally, Actis (2000) states that physical properties of the tailings dams can be determined by friction and/ or cohesion laboratory testing, and by drained, undrained, slow, quick, and consolidated triaxial and direct shear tests, considering dam operating conditions.

Tailings dam No. 3 of the Mahr Túnel benefit concession is located in the Yauli district and Province, Department of Junín- Peru at an elevation of $4000 \mathrm{MASL}$, where weather is cold due to altitude. The area experiences mostly rainy weather, with maximum precipitation between November and April, as well as persistent snow and hailstorms. The annual maximum precipitation is 1188 $\mathrm{mm}$ during the wet season and $576 \mathrm{~mm}$ during the dry season (May-November). Furthermore, maximum temperatures can reach $17{ }^{\circ} \mathrm{C}$, dropping to $-13^{\circ} \mathrm{C}$ in July and falling to near $0^{\circ} \mathrm{C}$ between September and December. The average temperature is $5.7^{\circ} \mathrm{C}$ according to the information obtained from the meteorological station at Pachachaca, at 4000 MASL.

Bachiller* in Civil Engineering. Independent consultant, supervisor of civil works and mining projects related to geotechnical engineering at the Ministry of Energy and Mines. Lima, Peru. E-mail: fmendoza@minem.gob.pe

*In Peru, Bachiller is the first academic degree awarded automatically after five years of university studies. 
It should be mentioned that dam No. 3 has been exposed to meteorological conditions that have caused regular changes in temperature, freezing of water and variations in tailings mineralogy. Similarly, such phenomena have provoked changes in the physical and chemical composition of the dam, resulting in a variation of the tailings properties and, consequently, its physical stability.

The foregoing situation has been studied by several researchers such as Ormazábal (2008), who conducted a study of time effects assessment (aging) on the static and dynamic behavior of tailings sand taken from the Ovejería dam (located at La Rinconada de Ovejería, at $550 \mathrm{MASL}$ ) in the Metropolitan region, Chacabuco Province, Chile.

Regarding the influence of meteorological causes, Joshi, Achari, Kaniraj and Wijiweera (1995) conducted an investigation by means of laboratory testing in order to study and record the effects of aging on sand, in addition to reviewing the influence of sand types and fluid in soil pores. Likewise, Richefeu, El Youssoufi and Radjai (2006) examined the shear strength properties of wet granular materials in the pendular state (discontinuous liquid phase) as a function of water content. Sand and sampling jars were moistened and tested in a direct shear cell under various confining pressures.

\section{Research Justification}

This study aims to more accurately determine the physical stability conditions of the aforementioned dam. To this end, geotechnical parameters of the dam filling material have been studied, considering their variation over time by the effects of meteorological conditions Thus, as per the factors of safety (FoS) obtained from the slope stability analysis, it was possible to determine the safety conditions and environmental actions that should be implemented in the area.

\section{Research Questions}

\subsection{General Research Question}

How to assess the shear strength behavior of the dam filling material, comprised of underflow tailings, altered by meteorological causes and aging, and its influence on slope stability of tailings dam No. 3 ?

\subsection{Specific Research Question}

In accordance with the framework of geotechnical field investigations, what type of sampling and soil mechanics testing are appropriate to estimate the variation of shear strength parameters of the dam in-fill material, comprised of underflow tailings, al- tered by meteorological causes, and how does it influence in the slope stability of tailings dam No. 3 ?

\section{Research Objectives}

\subsection{General Objective}

Assess the shear strength behavior of the dam in-fill material, comprised of underflow tailings, considering the meteorological causes and possible effects on the physical stability of tailings dam No. 3 .

\section{2.Specific Objective}

Determine the shear strength variation of the dam in-fill material, comprised of underflow tailings. Evaluate said variation through triaxial testing of undisturbed samples, which are representative samples of material exposed to meteorological conditions and establish the influence of meteorological conditions on the slope stability of tailings dam No. 3 .

\section{METHODOLOGY}

\section{Collection and analysis of existing data}

This consisted of the collection and analysis of data regarding site conditions, engineering designs, construction and operational reports of tailings dam No. 3, provided by Volcan Compañía Minera S.A.A. Additionally, information regarding the storage facility permits was obtained from the Ministry of Energy and Mines of Peru.

\section{Field investigation and laboratory testing}

\subsection{Geotechnical investigation}

A program of field and laboratory geotechnical investigations was conducted, and data from previous studies was also used. Boreholes with test pits, conveniently located and distributed within tailings dam No. 3 were carried out in order to obtain undisturbed samples of tailings. Following a detailed description of the material, undisturbed samples were taken, which were identified and protected in order to be sent to the soil mechanics laboratory.

\subsection{Field geotechnical investigation}

To obtain tailings samples that reflect the actual disposal conditions before meteorological conditions, undisturbed samples from the dam were analyzed and three test pits located and distributed within the tailings dam were carried out. In order to obtain undisturbed tailings samples, according to the depth recorded by the test pit, fresh, moist tailings samples were considered. Then, the surface was prepared by marking the outline of the upper face of the sample. Figures 1 and 2 show the undisturbed samples. 
To complement this information, data on the geotechnical boreholes conducted during the construction and operations stages of the tailings storage facility was used.

\section{Laboratory testing}

In order to demonstrate the real conditions of the tailings material, a consolidated undrained (CU) triaxial test with pore pressure measurement was conducted on the sample. In this way, these tests can compensate for the effects of disturbance resulting from the extraction of the undisturbed samples. In addition, available reports on triaxial testing with similar characteristics to those of the sample type and confining pressures were considered. Selected prototypes were compressed using confining stress values $(\sigma c)$ of $1.0,2.0$ and $4.0 \mathrm{~kg} / \mathrm{cm}^{2}$. The testing was conducted on the soil mechanics laboratory of the Pontificia Universidad Católica del Perú (PUCP). Likewise, index testing (physical properties) were conducted to identify and classify the material as per the Unified Soil Classification System (USCS). The triaxial test consisted in preparing soil specimens wrapped with a membrane used to drain and maintain control of pore pressure within the sample; therefore, porous stones were placed at the base and the upper part of each sample (González de Vallejo, Ferrer, Ortuño \& Oteo, 2002).

\subsection{Office research}

- Estimation of the age of existing and recent undisturbed samples boreholes, based on topographic information and geotechnical boreholes conducted in various design studies for the raising of tailings dam No. 3 , from 2007 to 2016.

- Production of borehole distribution plan view drawings and projection of the periods of exposure to meteorological conditions.

- Analysis, interpretation and discussion of the results obtained from both the $\mathrm{CU}$ triaxial testing and the standard testing of the different samples, according to the period of exposure to meteorological conditions.

- Estimation of physical and shear strength of underflow tailings parameters based on laboratory reports.



Figure 1. Sample extraction and paraffin coating. Source: Prepared by the author.



Figure 2. Packing and transport of a $30 \times 30 \times 30 \mathrm{~cm} 3$ undisturbed tailings sample.

Source: Prepared by the author. 
- Geotechnical model determination based on different materials and geometry parameters defined for storage periods.

- Stability analysis of the tailings dam according to the geotechnical model designed for the initial stage and for different periods of meteorological exposure.

\section{RESULTS}

Considering the objectives of this study, geotechnical studies regarding the undisturbed tailings samples were conducted in order to determine their behavior against meteorological conditions over time. The results are described below.

\section{Estimation of periods of tailings disposal}

Figure 3 shows the age estimation of undisturbed samples obtained from three test pits. The diagram was produced based on topographic information from the dam raise and the period of tailings disposal.

\section{Particle size distribution of tailings}

According to the index tests and the USCS, underflow tailings classifies as silty sands (SM) and silty-clayey sands (SM-SC). The sand content (\#+200) ranges between $57 \%$ and $83 \%$, whereas fines content (\#-200) ranges between 16\% and $42 \%$.

\section{Shear strength variation based on time of exposure to meteorological conditions}

The triaxial testing results, in terms of total stress, indicate that when weathering time (disposal) is increased, undrained shear strength "increases" as well. This indicates that time of exposure to meteorological conditions influence the increase on tailings cohesion and friction angle.

Figure 4 shows the comparison friction angles results. Thus, sample BH4-SMR3 has an estimated disposal period of three (3) years, increased by $60 \%$ compared to the initial disposal year "zero" (0). It is followed by an increasing trend until disposal year six (6). An increase of up to $200 \%$ is observed in sample C-2 M-1. Meanwhile, a decrease of up to $68 \%$ regarding year seven (7) compared to year zero $(0)$ is observed for sample BH5-SMR3.

Figure 5 shows the comparison of the cohesion results. For year zero (0), a cohesion value equal to $0 \mathrm{~kg} / \mathrm{cm}^{2}$ is observed in sample SPT CM-11; whereas a sudden increase that reaches cohesion values equal to $230 \mathrm{~kg} / \mathrm{cm}^{2}$ is observed in sample BH4SMR3, with three (3) years of disposal. However,

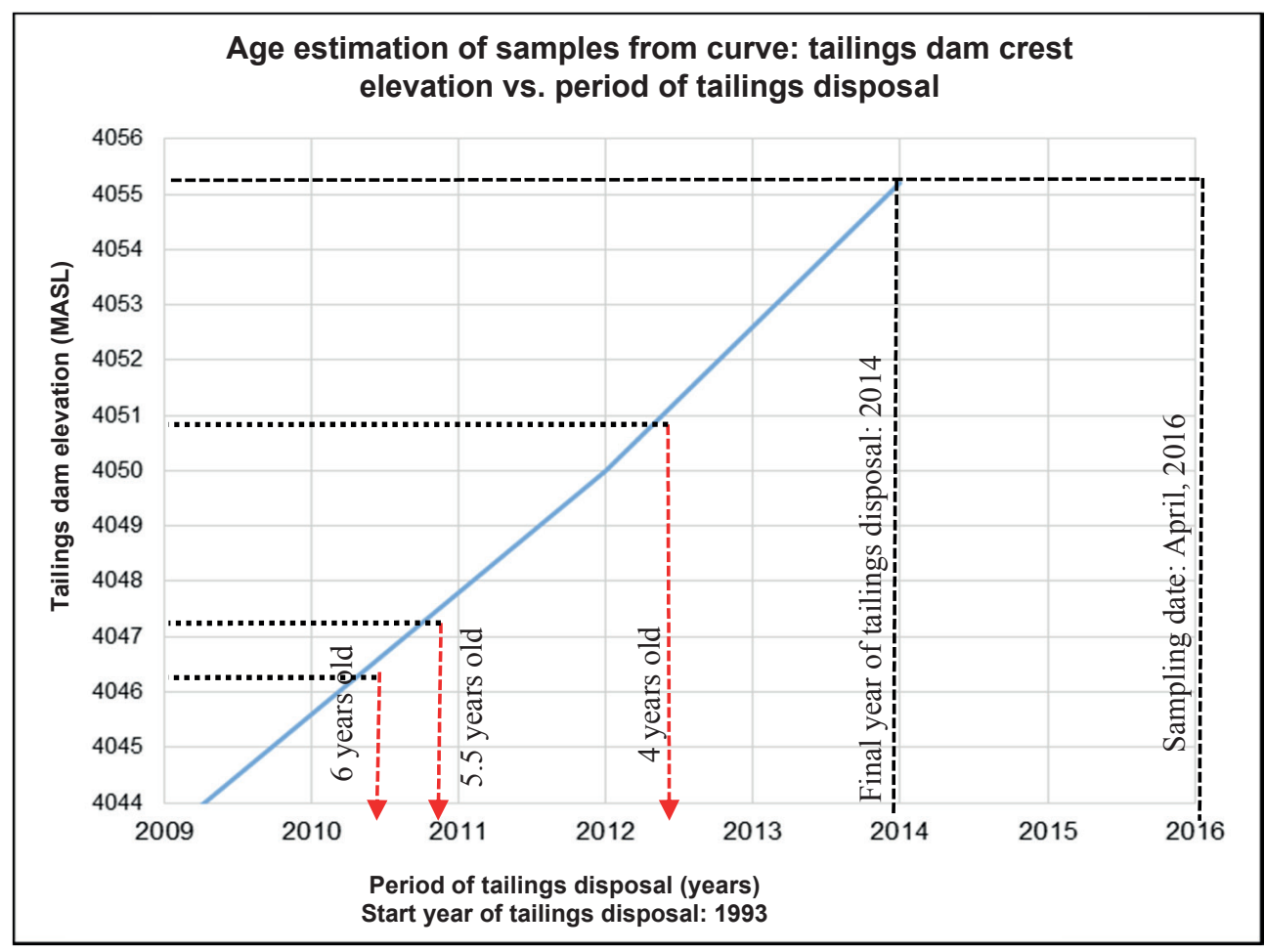

Figure 3. Age estimation of samples based on the curve: tailings dam crest elevation versus period of tailings disposal

Source: Prepared by the author. 
a decrease that reached cohesion values of $78 \mathrm{~kg} /$ $\mathrm{cm}^{2}$ is observed in sample C-1 M-1 for year four (4). A progressive increase in cohesion is expected for the following years.

\section{Slope stability analysis}

\subsection{General}

The following was considered for the slope stability analysis of tailings dam No. 3 :

- A cross-section analysis based on the current geometric configuration of the dam and boreholes was developed, which was used to estimate the distribution of materials.
- The cross-section represents the dam's critical conditions, that is, the greatest slope angle, seismic load, water table conditions, among others.

- The limit equilibrium method was used to assess the slope sliding resistance, considering certain hypotheses regarding failure mechanisms, equilibrium conditions, water table surface, shear strength parameters, etc.

\subsection{Analysis methodology}

- SLIDE software (Rocscience version 6) was used to interactively model the slope geometric configuration as well as



Figure 4. Shear strength variation of underflow tailings friction angle in terms of total stress. Source: Prepared by the author.



Figure 5. Variation of underflow tailings cohesion regarding total stress. Source: Prepared by the author. 
to define different materials, considering their geotechnical properties (physical and mechanical).

- The determination of the factors of safety (FoS) was based on the work conducted by Quispe (2015), who notes that it is "se lleva a cabo de manera bidimensional usando el concepto de equilibrio límite, aproximando el problema a un estado de deformación plana" [conducted in a two-dimensional way following the concept of the limit equilibrium, approaching the problem to a state of plane strain] (p. 4). Furthermore, this author states that the critical failure surface is the one that possesses the lowest FoS, the same that is found interactively by modifying failure surface search conditions as well as by evaluating circular slip surfaces.

- At the same time, as hypothesis of the stability analysis, "se considera que las propiedades [físicas y mecánicas] de los materiales que conforman las estructuras analizadas son homogéneas e isotrópicas y que el colapso se produciría como resultado de fallas simultáneas a lo largo de la superficie de deslizamiento" [considers that the properties [physical and mechanical] of materials that comprise the analyzed structures are homogeneous and isotropic, and that collapse would occur as a result of simultaneous failures along the sliding surface] (Quispe, 2015, pp. 4-5). The pseudo-static stability analysis subjects a two-dimension sliding mass to a horizontal acceleration equal to the seismic coefficient multiplied by the gravity acceleration.
- The method proposed by Hynes-Griffin and Franklin (1984) is used for the pseudostatic stability analysis, which requires the reduction of shear strength properties of material up to $20 \%$ for materials subjected to a pore pressure water increase under cyclic conditions (earthquakes).

\subsection{Design seismic factor}

The design parameter specified for the study area is a peak ground acceleration of $0.3 \mathrm{~g}$, with a $10 \%$ exceedance probability in 50 years of exposure to seismic events, corresponding to a 475-year return period. The recommended coefficient of stability under pseudo-static conditions ranges between 1/2 and $1 / 3$ times the peak ground acceleration value. In their study, Rennat and Miller (1997) used a seismic coefficient of $0.15 \mathrm{~g}$, which is equal to $1 / 2$ of the expected peak ground acceleration of $0.3 \mathrm{~g}$.

\subsection{Water table}

Water table depths are between $16.20 \mathrm{~m}$ and 14.60 $\mathrm{m}$ from the soil surface. These water levels act as a piezometric level, as they mainly affect foundation soil and bedrock, which constitutes a conservative hypothesis since water level is not continuous. In fact, Rodríguez and Oldecop (2011) state that water from seepage within the tailings dam quickly saturates pores and partially fills them with capillary water. In addition, the proximity of water table to the dam's outer slope has destabilizing effects.

\section{Geotechnical parameters}

Table 1 shows the geotechnical properties of the different materials used in the slope stability analysis. These shear strength parameters resulted from the CU triaxial testing on undisturbed and disturbed samples.

Table 1. Summary of geotechnical properties of materials.

\begin{tabular}{|c|c|c|c|c|c|c|c|}
\hline \multirow{2}{*}{ Material } & \multirow{2}{*}{$\begin{array}{c}\text { Disposal } \\
\text { year }\end{array}$} & \multirow{2}{*}{$\begin{array}{c}Y_{\text {sec }}(\mathrm{kNN} / \\
\mathrm{m})\end{array}$} & \multirow{2}{*}{$\begin{array}{c}\mathrm{Y}_{\text {sat }}(\mathrm{kN} / \\
\mathrm{m})\end{array}$} & \multicolumn{2}{|c|}{ Total } & \multicolumn{2}{|c|}{ Effective } \\
\hline & & & & $\mathrm{C}(\mathrm{kPa})$ & $\Phi\left({ }^{\circ}\right)$ & $\mathrm{C}(\mathrm{kPa})$ & $\varphi\left({ }^{\circ}\right)$ \\
\hline Remolded underflow tailings sample (C11, M-1) & 0 & 16 & 17.5 & 0 & 16 & 30 & 31.5 \\
\hline Underflow tailings (BH4-SMR3-04) & 3 & 21 & 24 & 230 & 26 & 0 & 41 \\
\hline Underflow tailings (C-1,M-1) & 4 & 19 & 21 & 78 & 35 & 50 & 35 \\
\hline Underflow tailings $(\mathrm{C}-2, \mathrm{M}-1)$ & 6 & 18.5 & 20 & 90 & 51 & 120 & 52 \\
\hline Underflow tailings (BH5-SMR3-04) & 7 & 17 & 24 & 120 & 27 & 29 & 36.4 \\
\hline Overflow tailings $\left({ }^{*}\right)$ & - & 16.5 & 20.0 & 0 & 25 & - & - \\
\hline Foundation & - & 19 & 20 & 100 & 36 & 10 & 36 \\
\hline
\end{tabular}

$\gamma_{\text {tot }}$ : total unit weight; $\gamma_{\text {Sat: }}$ saturated unit weight; $C$ : cohesion; $\varphi$ : internal friction angle.

$\left(^{*}\right)$ Value estimated from previous studies.

Source: Prepared by the author. 


\section{Results of the slope stability analyses}

The dam stability analyses were performed considering underflow tailings not exposed to meteorological conditions, year zero, and underflow tailings exposed to meteorological conditions. For the first case, FoS values of 1.014 were obtained under pseudo-static conditions (see Figure 6). For the second case, FoS values of 1.060 were obtained under pseudo-static conditions (see Figure 7).

\section{DISCUSSION}

\section{Particle size distribution of tailings}

Peters (2004) indicates that the particle size distribution of tailings reflects the treatment process used to obtain the ore, the placement system and the disposal method. Beach slope towards the reservoir where overflow tailings are deposited ranges between $0.5 \%$ and $3.0 \%$ in the first $30 \mathrm{~m}$, then decreases up to $0.1 \%$ in the proximity of the pond. In the case of underflow tailings, average slopes can reach $4 \%$ and $6 \%$. In terms of size, tailings are generally bigger than $0.074 \mathrm{~mm}$, which means that less than $50 \%$ of them are smaller than said size. In addition, lamas is "el material predominantemente del tamaño del limo, por lo cual, más del $50 \%$ es menor que $0,074 \mathrm{~mm}$ " [the material predominantly of the size of silt, therefore, less than $50 \%$ is smaller than $0.074 \mathrm{~mm}$ ] (Torres, 2014, p. 42).

2. Variation of deviator stress and pore pressure with respect to the deformation of tailings samples with exposure periods of meteorological conditions.

Figure 8 shows the comparison between the deviator stress and strain results obtained for the various analyzed samples, with a chamber pressure of $4 \mathrm{~kg} /$

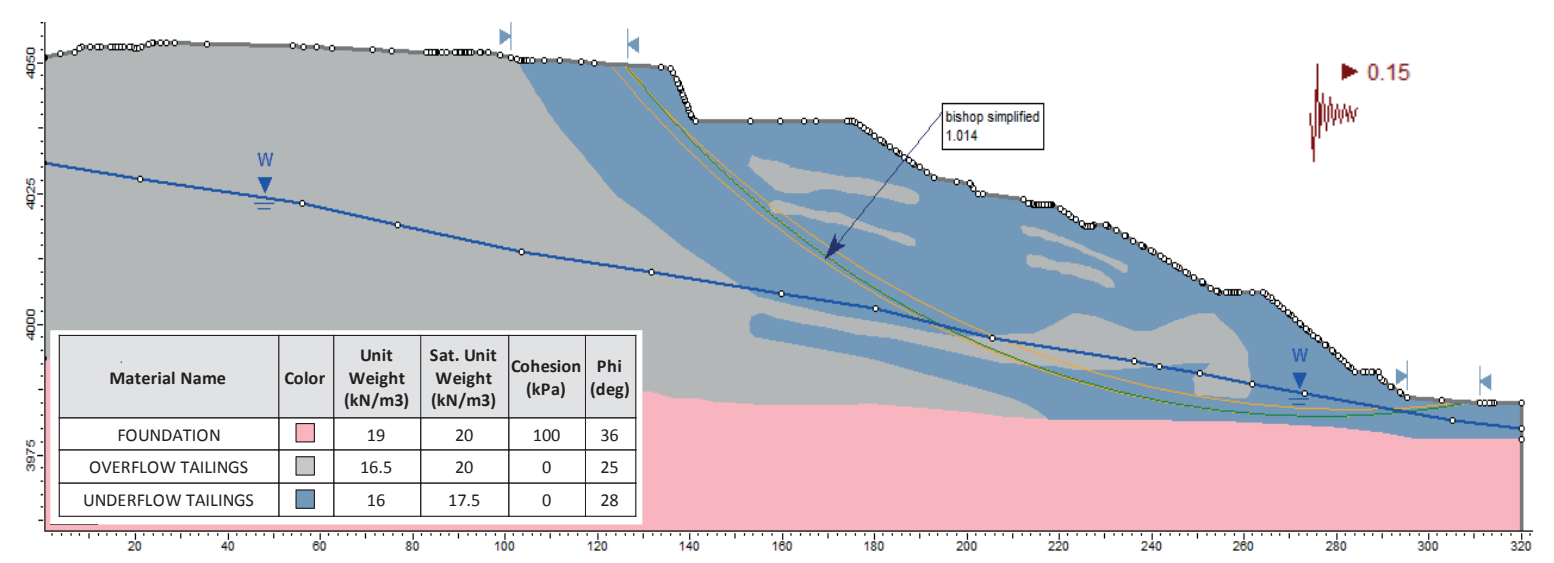

Figure 6. Tailings dam No. 3 stability analysis. Pseudo-static slope stability with no exposure to meteorological conditions (year 0), circular slip surface.

Source: Prepared by the author.

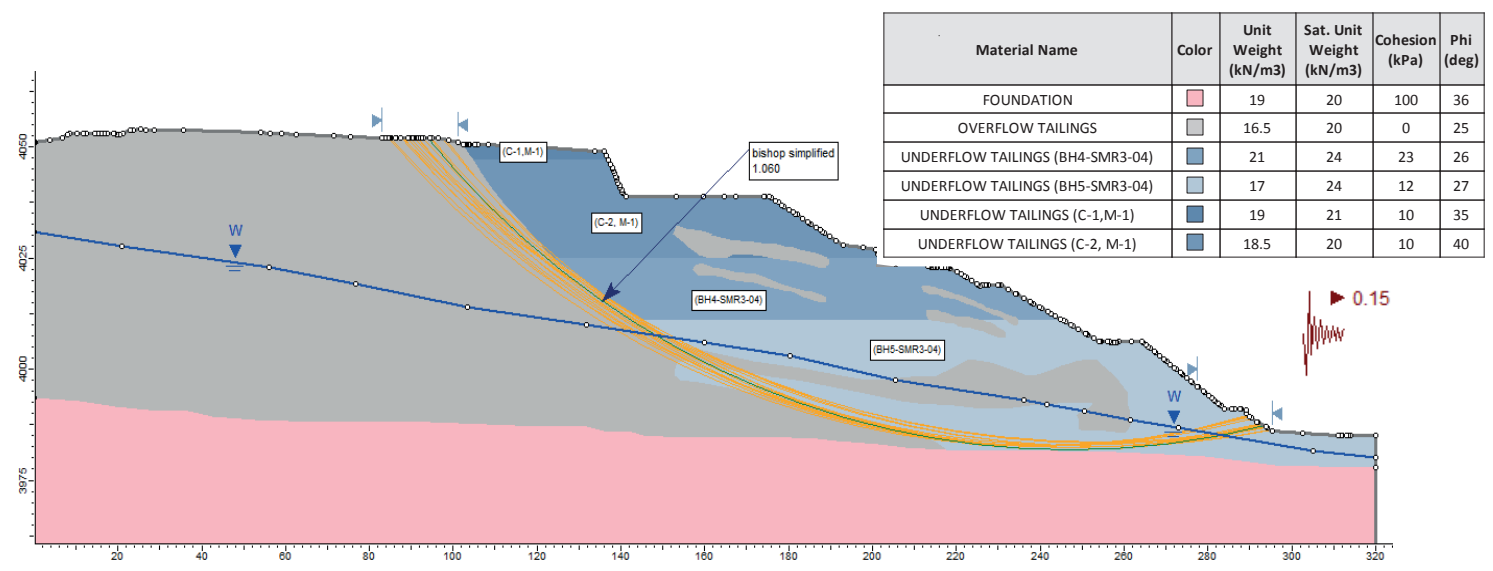

Figure 7. Tailings dam No. 3 stability analysis. Pseudo-static slope stability with exposure to meteorological conditions, circular slip surface.

Source: Prepared by the author. 


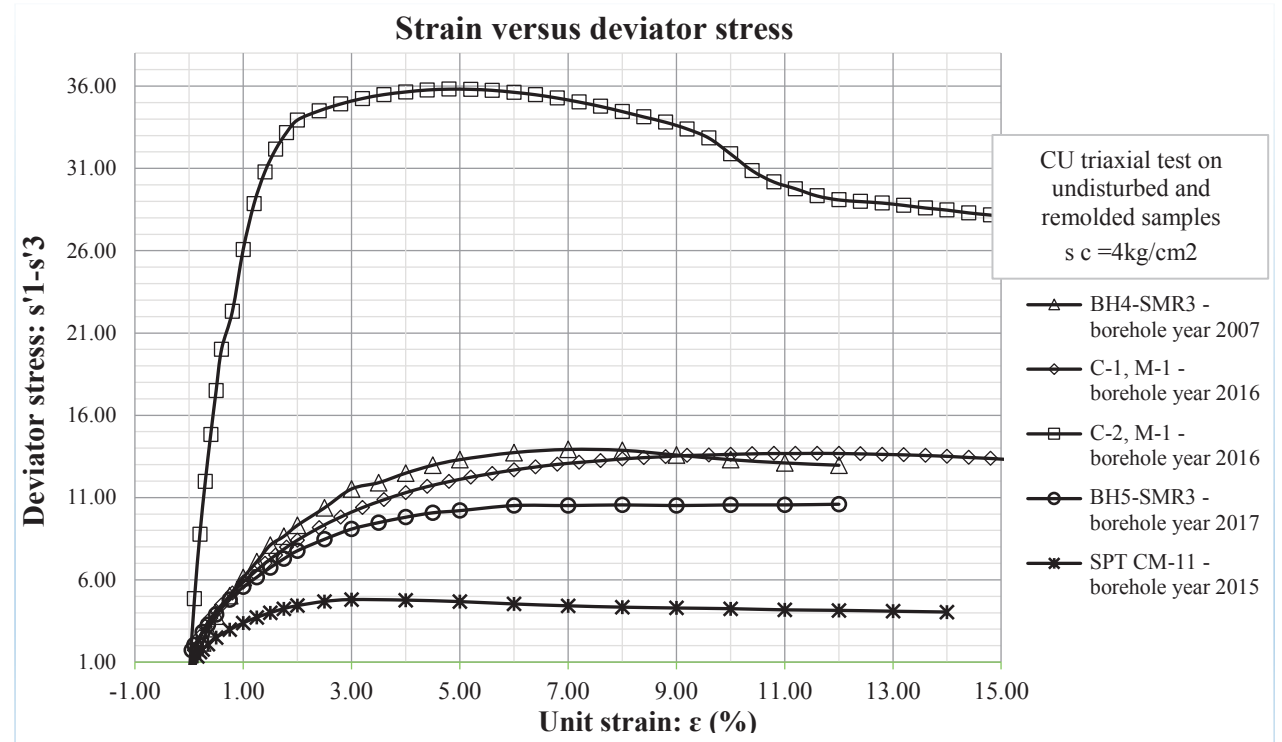

Figure 8. Strain $(\varepsilon)$ versus deviator stress of undisturbed samples tested in different periods and the remolded sample.

Source: Prepared by the author.

$\mathrm{cm}^{2}$, considering the estimated number of years that tailings were exposed to meteorological conditions in dam No.3.

A fragile-type failure is observed for the (disturbed) sample representing year zero, because, as stress increases, strain increases and tends to have a limit value that remains even though strain continues to increase to very high values. For undisturbed samples (years 3, 4, 6 and 7 of tailings disposal), strain increases with regard to year zero, which corresponds to a fragile-type failure, that is, when stress reaches a maximum value, it decreases as strain increases, but as strain increases, the last value tends to be the same. For the sample representing year 6 (C-2 M-1), the stress-strain curve corresponds to cemented sand, in other words, the rapid decrease of stress from the maximum value occurs when strain increases.

Figure 9 shows the applied chamber pressure $\sigma_{3}$ for each undisturbed and remolded sample. Shear strength is initially withstood by water, and then pore pressure within the tailings increases. For remolded sample SPT CM-11, water pressure withstands tailings shear strength at all times. Pore pressure becomes negative in sample BH4-SMR3 at a strain of $10.0 \%$; in sample $\mathrm{C}-1 \mathrm{M}-1$ at a strain of $6.5 \%$; and in sample C-2 M-1 at a strain of $2.0 \%$. This decrease and change in pore pressure indicates that tailings particles withstand the load and that tailings effective stress is increasing.

\section{Variation of tailings density with meteorological exposure periods and their influence on shear strength parameters}

Based on the studied samples of different disposal periods of underflow tailings, density in situ for year 0 is $1.77 \mathrm{~g} / \mathrm{cm}^{3}$; for year $3,2.33 \mathrm{~g} / \mathrm{cm}^{3}$; for year $4,2.18 \mathrm{~g} /$ $\mathrm{cm}^{3}$; for year $6,2.02 \mathrm{~g} / \mathrm{cm}^{3}$ and for year $7,2.06 \mathrm{~g} / \mathrm{cm}^{3}$. As indicated, initial density (year 0 ) is low; however, densities increase as disposal years pass. The same occurs to shear strength parameters, which increase due to secondary consolidation. In this regard, Badillo and Rodríguez (2005) state that secondary consolidation is generally attributed to the progressive creep among material particles that are rearranged until a more compact state to adapt to the new load is obtained. Thus, the degree of consolidation significantly influences tailings properties, rendering tailings less deformable, denser and more resistant. In addition, variations in dry density are more noticeable during the consolidation process with the porosity rate decrease, which leads to the formation of more compact and rigid structures. In some cases, mine tailings present significant secondary consolidation (no increase in vertical load), either because particle disaggregation or due to creep (Ayala \& Rodríguez, 1986).

\section{Factors of safety from slope stability analysis}

According to the results obtained from the dam stability analysis, factors of safety for tailings dam 


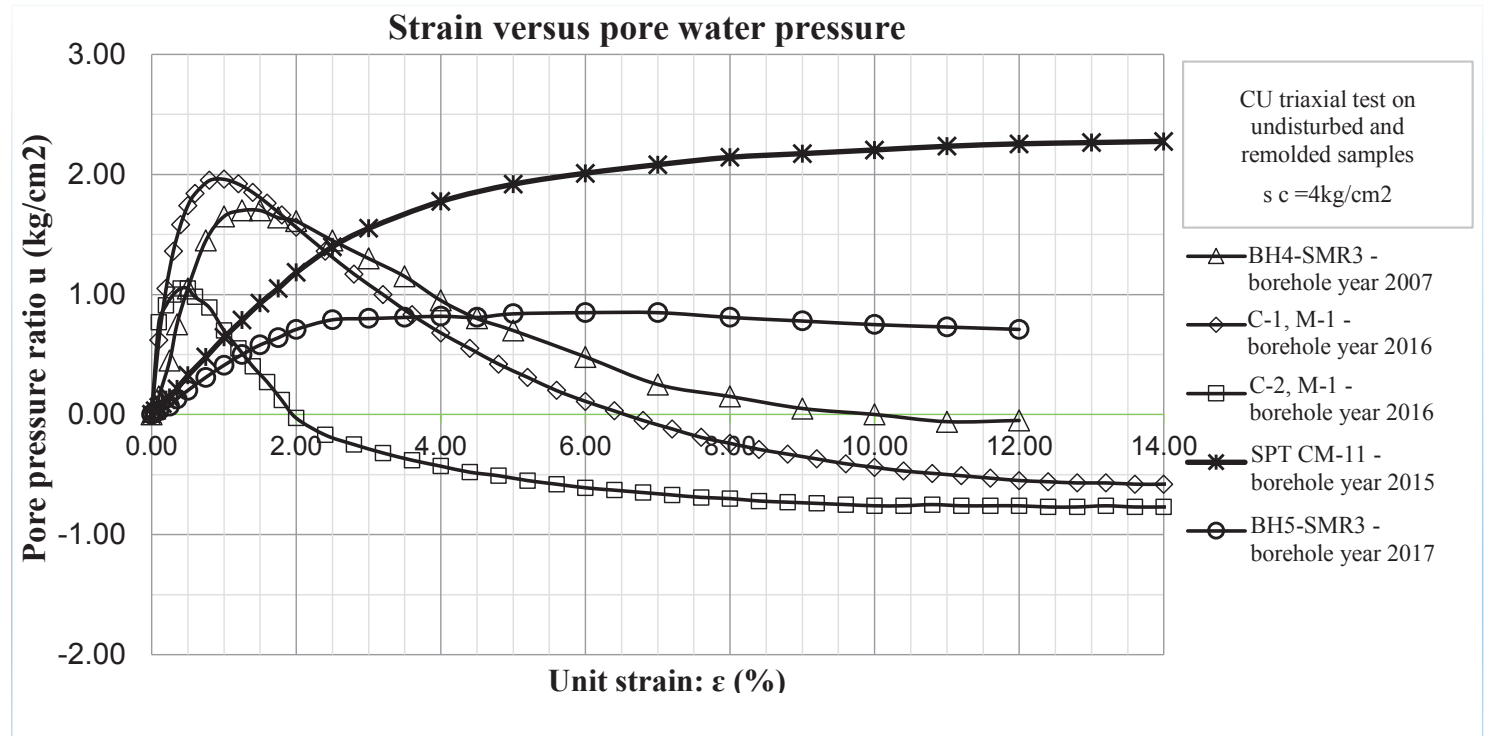

Figure 9. Unit strain $(\varepsilon)$ versus pore pressure $(u)$ of undisturbed samples tested in different periods and the remolded sample.

Source: Prepared by the author.

No. 3, with tailings material exposed to weathering, are more stable in pseudo-static conditions.

\section{CONCLUSIONS}

- Shear strength behavior of in-fill material comprised of underflow tailings was evaluated considering meteorological causes and their possible effects on the physical stability of tailings dam No. 3. To this end, geotechnical boreholes were conducted to obtain undisturbed tailings material samples using standardized procedures. After samples extraction, consolidated undrained triaxial testing with pore pressure measurement was conducted and validated. Said testing reflected the stress-strain and soil strength characteristics as a result of the tailings dam critical conditions, effects of disturbance of the undisturbed sample and, consequently, of the meteorological effects.

- In terms of total stress and effective stress, the friction angles results $(\varphi)$ obtained for underflow tailings from tailings dam No. 3 exposed to different periods of weathering were as follows: $35^{\circ}$ for year 4 , and $52^{\circ}$ and $51^{\circ}$ for year 6 . According to previous data, the friction angles results for underflow tailings not exposed to meteorological conditions were $16.5^{\circ}$ and $31.5^{\circ}$ for year $0 ; 26^{\circ}$ and $41.4^{\circ}$ for year 3 ; and $27^{\circ}$ and $36.4^{\circ}$ for year
7. These results indicate that the friction angle increased as the period of exposure to meteorological conditions increased.

- In terms of total stress and effective stress, the cohesion (c) results obtained for underflow tailings under different periods of exposure to meteorological conditions were as follows: $50 \mathrm{kPa}$ and $78 \mathrm{kPa}$ for year 4, and $6.90 \mathrm{kPa}$ and $120 \mathrm{kPa}$ for year 6 . According to previous data regarding the tailings dam No. 3 , the cohesion results obtained were $0 \mathrm{kPa}$ and 3 $\mathrm{kPa}$ for year 0; $230 \mathrm{kPa}$ and $0 \mathrm{kPa}$ for year 3 ; and $120 \mathrm{kPa}$ and $29 \mathrm{kPa}$ for year 7. These results indicate that cohesion increased as the period of exposure to meteorological conditions increased.

- The following results were obtained for in situ density: $1.77 \mathrm{~g} / \mathrm{cm}^{3}$ for year $0 ; 2.33 \mathrm{~g} / \mathrm{cm}^{3}$ for year $3 ; 2.02 \mathrm{~g} / \mathrm{cm}^{3}$ for year 6 ; and 2.06 $\mathrm{g} / \mathrm{cm}^{3}$ for year 7 . In other words, density is low for the initial year and it increases as the years of exposure to meteorological conditions increase; therefore, shear strength parameters result from secondary consolidation. For this reason, Das (2013) states that secondary consolidation of soil occurs after the total dissipation of excess pore water pressure, after the sample experiences straining as a result of the plastic adjustment of soil grains. 
- Based on the cohesion increase in basically cohesive materials present in the tested tailings (sand with an average of $21.99 \%$ fines content, namely, percentage of fines passing sieve \#200), the increase of cohesion was determined. This resulted in a progressive cementing of material due to the mineralogical composition of tailings, provoking intergranular pressure for the effects of capillarity, additionally creating an "apparent cohesion". It should be noted that, according to Lambe and Whitman (1991), apparent cohesion is lost as soon as the soil is submerged below the water table.

- A factor of safety of 1.014 in pseudo-static condition was obtained for underflow tailings not exposed to meteorological conditions, and a factor of safety of 1.060 in pseudo-static condition was obtained for tailings exposed to meteorological conditions. Thus, factors of safety of tailings exposed to meteorological conditions are greater than those not exposed to them. These values indicate that tailings dam No. 3, with tailings material exposed to weathering, has more stability under pseudostatic conditions.

\section{ACKNOWLEDGEMENTS}

To Enrique Guadalupe Gómez, Victor Abel Tolentino Yparraguirre and Jorge Escalante Contreras, professors at the Universidad Nacional Mayor de San Marcos, for their continued support in their careful review of the paper. Likewise, to Volcan Compañía Minera S. A. A., for making its facilities available for this study.

\section{REFERENCES}

[1] Ayala, F. \& Rodríguez, J. (1986). Manual para el diseño y construcción de escombreras y presas de residuos mineros. Madrid, Spain: Instituto Geológico y Minero de España.

[2] Actis, R. A. (2000). Diques de colas mineras. Cálculo, diseño, construcción y operación. Córdoba, Argentina: Fundación EMPREMIN.

[3] Badillo, J. \& Rodríguez, R. (2005). Fundamentos de Mecánica de Suelos (tomo 1). Mexico D. F., Mexico: Limusa Noriega Editores.

[4] Das, B. M. (2013). Fundamentos de ingeniería geotécnica. Mexico D. F., Mexico: Cengage Learning Latinoamérica.
[5] González de Vallejo L., Ferrer M., Ortuño L. \& Oteo, C. (2002). Ingeniería geológica. Madrid, Spain: Pearson Education.

[6] Hynes-Griffin, M. E. \& Franklin, A. G. (1984), Rationalizing the Seismic Coefficient Method. Retrieved from http://acwc.sdp.sirsi. net/client/en_US/default/index.assetbox. assetactionicon.view/1003264

[7] Joshi, R. C., Achari, G. Kaniraj, S. R. \& Wijiweera, H. (1995). Effect of aging on the penetration resistance of sands. Canadian Geotechnical Journal, 32(5), 767-782.

[8] Lambe, T. W. \& Whitman, R. V. (1991). Mecánica de fluidos. Mexico D. F., Mexico: Limusa Noriega Editores.

[9] Ormazábal, M. G. (2008). Estudio en laboratorio del efecto envejecimiento en el comportamiento de arenas de relave. (Undergraduate Thesis). Universidad de Chile, Santiago de Chile. Retrieved from http://repositorio.uchile.cl/ bitstream/handle/2250/104855/ormazabal_ $\mathrm{ml}$.pdf? sequence $=3$ \&isAllowed $=y$

[10] Peters, G. (2004). Propiedades geotécnicas de arenas de relave y sus implicancias en el diseño de tranques. En E. Riesco (Presidencia), $\checkmark$ Congreso Chileno de Ingeniería Geotecnia. Conferencia llevada a cabo en la Universidad de Chile, Santiago de Chile.

[11] Quispe, A. (2015). Factor de seguridad en la estabilidad en pad de lixiviación (Undergraduate Thesis). Universidad Nacional de Ingeniería, Lima. Retrieved from http://cybertesis.uni.edu. pe/handle/uni/5606

[12] Rodríguez, R. \& Oldecop, L. (2011). Humedad y estabilidad geotécnica de presa de relaves. Retrieved from https://www.researchgate. net/publication/306292392_Humedad_y_ estabilidad_geotecnica_de_presas_de_ relaves.

[13] Rico, M., Benito, G., Salgueiro, A., et al. (2007). Reported tailing dam failures: A review of the European incidents in the worldwide context. Journal of Hazardous Materials, 152(2), 846852.

[14] Rennat, E. \& Miller, S. (1997). Guía ambiental para la estabilidad de taludes de depósitos de desechos sólidos de mina. Lima, Perú. Ministerio de Energía y Minas. Retrieved from http://www.minem.gob.pe/minem/archivos/file/ DGAAM/guias/guiaestabilidad.pdf 
[15] Richefeu, V., El Youssoufi, M. S. \& Radjaï, F. (2006). Shear strength properties of wet granular materials. Physical Review, (73), 1-12. Retrieved from https://www.researchgate. net/publication/6981623_Shear_strength_ properties_of_wet_granular_materials.
[16] Torres, E. (2014). Práctica supervisada. Proyecto de sistema de sedimentación para la compañía minera El Aguilar. Retrieved from https://rdu.unc.edu.ar/handle/11086/1533. 